\title{
Denker der neuropsychischen Ganzheit
}

\section{Niklaus Egloff}

PD Dr. med., Leiter Kompetenzbereich für Psychosomatische Medizin, Universitätsklinik für Neurologie, Inselspital Bern

Als Internist, Neurologe und Psychotherapeut fokussierte Paul Dubois auf die Behandlung von funktionellen Leiden. Wie Freud hatte er Zulauf von Patienten aus ganz Europa. Dubois setzte therapeutisch aber nicht auf das Unbewusste, sondern auf den Verstand und den Dialog. Seine medizinischen Hauptwerke sind Anfang des 20. Jahrhunderts in fast sämtliche europäischen Sprachen übersetzt worden. Als Experte für funktionelle Körpersymptomstörungen gilt Dubois als wichtiger Exponent der Geschichte der psychosomatischen Medizin.

\section{Medizinische Rezeption und Bedeutung}

1902 wurde Paul Dubois ausserordentlicher Professor für Neuropathologie an der Universität Bern. Dubois ist sowohl Mitbegründer der Schweizerischen $\mathrm{Ge}^{-}$ sellschaft für Neurologie wie auch Mitbegründer der Psychologischen Vereinigung von Bern. Als Mitherausgeber der 1917 erstmals erschienenen Fachzeitschrift Schweizerische Archive für Neurologie und Psychiatrie ist Dubois sowohl im Gedächtnis der schweizerischen Neurologie als auch der schweizerischen Psychiatrie ein Referenzpunkt. Aufgrund seiner Spezialisierung auf funktionelle Körpersymptomstörungen gilt Dubois als wichtiger Exponent der schweizerischen Geschichte der psychosomatischen Medizin.

Als eines seiner medizinischen Hauptwerke gilt das 1904 zunächst auf Französisch erschienene Standardwerk Die Psychoneurosen und ihre seelische Behandlung [1]. In 35 Vorlesungskapiteln referiert Dubois sein Verständnis von funktionellen Erkrankungen. Liest man

Dubois schaffte einen erstaunlich hohen Grad an differentialdiagnostischer Unterscheidung.

Dubois' medizinische Publikationen aus der Perspektive der heutigen Medizin, beeindrucken die differenzierten klinischen Beschreibungen und seine integrative Interpretationsweise. Er schaffte einen erstaunlich hohen Grad an differentialdiagnostischer Unterscheidung, ohne dass ihm all die technischen Untersuchungsmöglichkeiten zu Verfügung gestanden haben,

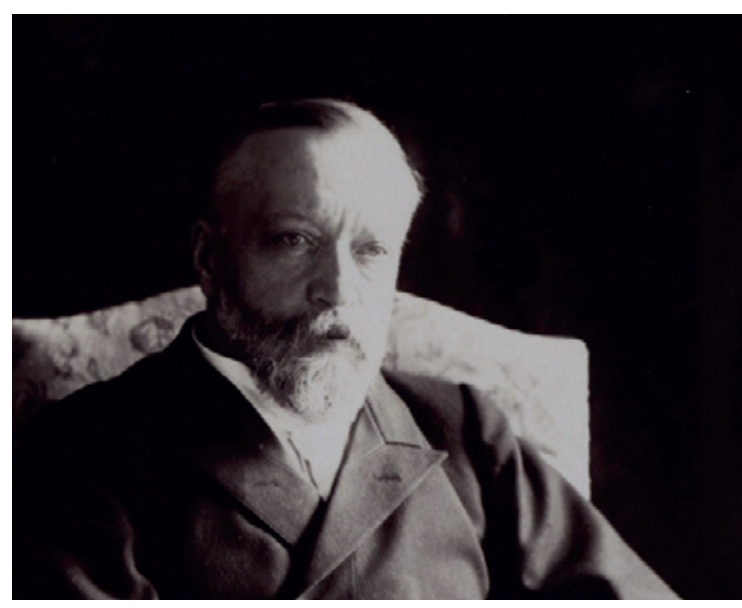

Paul Dubois (1848-1918).

wie wir sie heute haben. So beschreibt und benennt er u.a. exakt die Phänomenologie von Krankheiten, wie wir sie heute als funktionelle Verdauungsstörungen, Anorexia nervosa, Fatigue-Zustände, Panikstörung wie auch als komplexe posttraumatische neurofunktionelle Ausfallsstörungen verstehen [1]. In einem Fallbericht dokumentierte er bereits 1888 sehr differenziert die funktionelle Hemihypästhesie als Folge eines Verkehrsunfalles, wie sie erst 100 Jahre später wieder in den Fokus der Medizin gerät [2, 3]. Auch thematisiert er bereits die opioidinduzierte Hyperalgesie, wie sie erst in den letzten Jahren in der Schmerzmedizin zunehmend erkannt wird [4]. 
Zahlreiche Publikationen, Bücher, Zeitungsbeiträge und Korrespondenzen weisen auf ein engagiertes Leben hin. Seine Hauptwerke sind in diverse west- und osteuropäische Sprachen übersetzt worden. Den bedeutsamsten und reichhaltigsten Einblick in sein Gesamtwerk verschafft die 2001 erschienene Biographie

Seine Hauptwerke sind in diverse west- und osteuropäische Sprachen übersetzt worden.

von Prof. Christian Müller [5]. Ein reicher Nachlass von Dubois' Schriften ist im Institut für Medizingeschichte der Universität Bern erhalten.

\section{Psychotherapie bei funktionellen Körpersymptomstörungen}

Dubois gilt als Begründer einer eigenen "rationellen» Psychotherapieform [6]. Wie Freud räumte er dem ärztlichen Gespräch einen eminenten Stellenwert in der Humanmedizin ein. Einer stoischen Grundhaltung verpflichtet, war seine Psychotherapie auf die Logik, Affektkontrolle, Gelassenheit, Selbstformung und den Dialog ausgerichtet. In Abgrenzung $\mathrm{zu}$ den damals gängigen Psychotherapiemethoden (Hypnose, Suggestivtherapie und Psychoanalyse) propagierte Dubois ein symmetrischeres Gesprächsverhältnis. In einer Art sokratischen Vorgehensweise zielte seine "rationelle Psychotherapie» darauf ab, seine Patienten von dysfunktionalen Krankheitsvorstellungen zu befreien und in der Selbstwirksamkeit zu stärken. Aus heutiger Sicht zeigt seine Psychotherapie vorweg den Charakter einer psychoedukativen Ausrichtung [6].

\section{Vorläufer einer integrativen psycho- somatischen Denkweise}

Gemäss den heutigen S3-Leitlinien für die Behandlung funktioneller Störungen leidet jeder zweite bis fünfte Patient in der Praxis an funktionellen Körpersymptomen [7]. Die Mehrheit der funktionellen Körpersymptome erklärt sich physiologisch auf Ebene vegetativer Dysregulation und/oder gesteigerter Körperperzeption; beide Aspekte hat Dubois bereits beschrieben. Was vor 100 Jahren mit Neurasthenie bezeichnet worden war, hat viel Ähnlichkeit mit dem, was wir heute unter Stressfolgestörungen verstehen. Dubois' wissenschaftstheoretischer Standort vor 100 Jahren liegt hinsichtlich des Krankheitsmodells gewissermassen dort, wo wir heute stehen: Er sah es als Selbstverständlichkeit an, seine Patienten mit ihren diversen organischen, funktionellen und psychischen Symptomen in deren neuropsychischer Ganzheit zu verstehen und zu behandeln. Er verstand es, stressassoziierte Körpersymptome in einem personalisierten Kontext zu lesen und mit seiner «rationellen Psychotherapie» zu lindern. Den damaligen Denkgewohnheiten voraus, deutete Dubois funktionelle Störungsbilder weder innerhalb eines kartesianisch gespaltenen Körper-Psyche-Dualismus noch in einem psychosomatischen Symbolismus. Im Gegenteil wurde Dubois als Neurologe und Internist nicht müde, seine Annahme zu betonen, dass auch funktionelle oder mentale Vorgänge neurophysiologische Korrelate haben. Er gerät dabei aber nicht in ein positivistisches Fahrwasser, sondern misst den senso-

Er verstand es, stressassoziierte Körpersymptome in einem personalisierten Kontext $\mathrm{zu}$ lesen.

rischen, affektiven und mentalen Informationsprozessen eine eigenständige Qualität zu, welche sich u.a. durch Psychotherapie modulieren lässt.

Literatur

1 Dubois P. Les psychonévroses et leur traitement moral: Leçons faites à l'université de Berne. Paris: Masson; 1904. Deutsche Übersetzung: Die Psychoneurosen und ihre psychische Behandlung: Vorlesungen gehalten an der Universität Bern. Bern: Francke; 1905

2 Dubois P. Über traumatische Neurosen. Correspondenz-Blatt für Schweizer Aerzte. 1891;17:522-38

3 Egloff N, Maecker F, Stauber S, Sabbioni ME, Tunklova L, von Känel R. Nondermatomal somatosensory deficits in chronic pain patients: are they really hysterical? Pain. 2012;153(9):1847-51.

4 Dubois P. L'éducation de soi-même. Paris: Masson; 1908. Deutsche Übersetzung: Selbsterziehung. Bern: Francke; 1909.

5 Müller C. «Sie müssen an Ihre Heilung glauben!» Paul Dubois (1848-1918). Ein vergessener Pionier der Psychotherapie. Basel: Schwabe Verlag; 2001.

6 Ducommun C. Paul Dubois (1848-1918). Sa place dans l'histoire de la psychothérapie. Gesnerus - Schweizerische Zeitschrift für Geschichte der Medizin und Naturwissenschaften. 1984;41:61-99.

7 Haller H, Cramer H, Lauche R, Dobos G. Somatoforme Störungen und medizinisch unerklärbare Symptome in der Primärversorgung. Deutsches Ärzteblatt. 2015;112(16):279-87.

Bildnachweis

Archiv des Instituts für Medizingeschichte der Uni Bern, Nachlass Paul Dubois, Schachtel 15. 